\title{
Preconditioning the Pressure Tracking in Fluid Dynamics by Shape Hessian Information
}

\author{
K. Eppler • S. Schmidt • V. Schulz • C. Ilic
}

Published online: 13 January 2009

(C) Springer Science+Business Media, LLC 2009

\begin{abstract}
Potential flow pressure matching is a classical inverse design aerodynamic problem. The resulting loss of regularity during the optimization poses challenges for shape optimization with normal perturbation of the surface mesh nodes. Smoothness is not enforced by the parameterization but by a proper choice of the scalar product based on the shape Hessian, which is derived in local coordinates for starshaped domains. Significant parts of the Hessian are identified and combined with an aerodynamic panel solver. The resulting shape Hessian preconditioner is shown to lead to superior convergence properties of the resulting optimization method. Additionally, preconditioning gives the potential for level independent convergence.
\end{abstract}

Keywords Shape optimization - Aerodynamic optimization · Hadamard gradient . Shape Hessian · Preconditioning

\section{Introduction}

Aerodynamic shape optimization is a field, where numerous progress has been made in the past years. Especially the introduction of the adjoint method $[1,2]$ has reduced

\footnotetext{
K. Eppler

Technical University Dresden, Dresden, Germany

e-mail: Karsten.Eppler@mailbox.tu-dresden.de

S. Schmidt $(\bowtie)$. V. Schulz

University Trier, Trier, Germany

e-mail: Stephan.Schmidt@uni-trier.de

V. Schulz

e-mail: Volker.Schulz@uni-trier.de

C. Ilic

German Aerospace Center (DLR), Braunschweig, Germany

e-mail: caslav.ilic@dlr.de
} 
the numerical costs of the gradient computation considerably. Instead of a sensitivity analysis for each parameter defining the shape, the computation of the gradient has become independent of the number of design parameters by solving an adjoint equation. This in turn leads to a tendency of using as many design parameters as possible for the shape discretization, preferably every surface node of the mesh.

In practice, however, this often seems to cause very rough gradients and their application in a gradient based optimization algorithm causes the shape to deteriorate rather quickly. We will justify this more theoretically by the explicit Hadamard representation of the shape gradient. As a remedy, Jameson has introduced the notion of gradient or so called Sobolev smoothing, preventing this loss of regularity. The impression of rough gradients without additional smoothing (by parameterizations or gradient smoothing) comes from the fact, that often the terms gradient and derivative are mixed up. The derivative is an object from the dual space, which can be represented as the gradient by the use of a scalar product. The choice of the scalar product to be used is not trivial and should be done by taking the Hessian operator into account. Thus, a smoothed steepest descent method is the same as an approximative Newton method.

This makes a detailed analysis of the structure of the shape Hessian necessary. It turns out that the shape Hessian acts like a pseudodifferential operator on a given boundary variation of the shape. In the field of computational fluid dynamics, several authors-especially Arian and Ta'asan [3], Arian and Vatsa [4] - have studied the highest order terms of the shape Hessian using Fourier analysis, and others have studied shape Hessians in more general fields [5]. In this paper, we will use a similar procedure for finding some information on the structure of the highest order terms of the shape Hessian, but we will also use shape calculus to add appropriate lower order terms, which are invisible to the Fourier transform. Adding the lower order terms turns the smoothing of the gradient into a preconditioner. That means, instead of purely conserving the regularity of the shape, the lower order terms provide a significant performance increase and more stability with an increase in design parameters.

Our studies will be focused on the potential flow pressure fitting. The velocity of the fluid is thought of as the gradient of some potential. By Bernoulli's law, the pressure is then inverse proportional to the speed of the fluid. Historically, the matching of a given pressure distribution with a new aircraft design was a means to carry over known good flight characteristics from an old to a new design. However, even today the potential flow pressure tracking is still used in the industry. Since the potential flow is computationally rather inexpensive, it is still in use during the mesh-making process.

Note that pressure fitting was already proposed by e.g. Jameson [6] as a useful model ("prototype") problem for wing design of an aircraft.

\section{Modeling: Statement of the Optimization Problem}

The aim of this paper is to discuss, analyze, and approximate the structure of the shape Hessian for the following shape optimization problem:

$$
\min _{\left(u, \Gamma_{1}\right)} J\left(u, \Gamma_{1}\right):=\int_{\Gamma_{1}} \frac{1}{2}\left(\langle\nabla u, \tau\rangle-p_{0}\right)^{2} d \Gamma_{1},
$$


Fig. 1 The domain $\Omega$ and the boundaries $\Gamma_{1}, \Gamma_{2}$

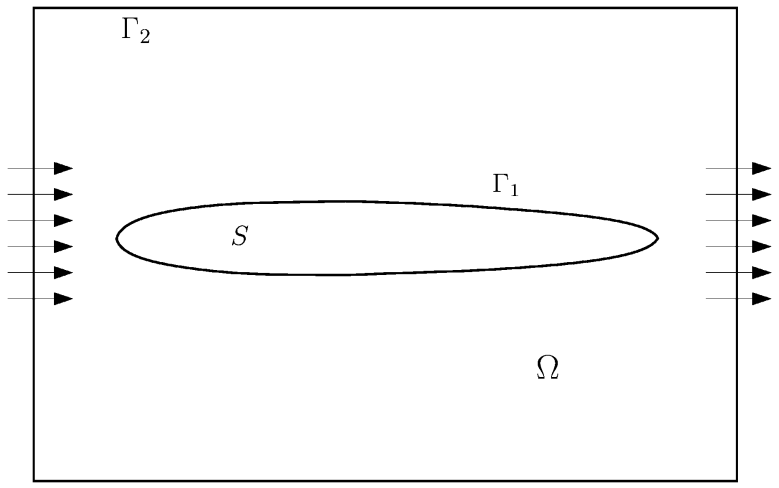

$$
\begin{aligned}
\text { s.t. }-\Delta u & =0, & & \text { in } \Omega, \\
\frac{\partial u}{\partial \vec{n}} & =0, & & \text { on } \Gamma_{1}, \\
u & =u_{0}, & & \text { on } \Gamma_{2},
\end{aligned}
$$

where $\Gamma_{1}$ denotes the boundary $\partial S$ of the airfoil $S$. Furthermore, $\Gamma_{2}$ is the farfield boundary where $u_{0}$ creates the necessary difference in the potential $u$ between inflow and outflow. Additionally, $p_{0}$ denotes the given pressure distribution to match. Finally, $\tau$ is the unit tangential vector to the $2 \mathrm{D}$ airfoil and $\langle\nabla u, \tau\rangle=: \frac{\partial u}{\partial \tau}$ is the tangential derivative of the potential, which is - when physically interpreted - the speed of the fluid in direction $\tau$. It is worth noting, that the unknown here is the complete boundary $\Gamma_{1}$ of the airfoil, see Fig. 1 . The question of existence of optimal shapes is not considered in this paper. Instead, we assume the existence of a regular optimal shape.

\section{Shape Calculus: First and Second Shape Derivatives}

\subsection{Hadamard Formulation of the Shape Gradient}

To provide a regular second order shape calculus, we assume the regularity $\Gamma_{1}, \Gamma_{2} \in$ $C^{3, \alpha}, u_{0} \in C^{3, \alpha}\left(\Gamma_{2}\right)$. The tracking data for the objective are given as a field $p_{0} \in$ $C^{2, \alpha}(\overline{\Omega \cup S})$. For a detailed discussion of spaces like $C^{2, \alpha}$ see [7].

For convenience, we recall classical results about the regularity of the state $u$.

Lemma 3.1 The regularity $u \in H^{2}(\Omega)$ is guaranteed for $\Gamma \in C^{2}$ and $u_{0} \in H^{3 / 2}\left(\Gamma_{2}\right)$. Moreover, the additional regularity $\Gamma_{1}, \Gamma_{2} \in C^{3, \alpha}, u_{0} \in C^{3, \alpha}\left(\Gamma_{2}\right)$ implies $u \in$ $C^{3, \alpha}(\bar{\Omega})$.

Of course, $u \in H^{2}(\Omega)$ already provides a meaning for the value $J\left(u, \Gamma_{1}\right)$. Nevertheless, the additional regularity is required for providing simultaneously regular boundary integral expressions for the shape gradient and the shape Hessian. 
Next, we derive the basic shape gradient formula in terms of a general domain perturbation approach, see also $[7,8]$ and the references therein for a general background on shape calculus.

Let $\mathbf{V} \in C^{3, \alpha}\left(\mathbb{R}^{n}, \mathbb{R}^{n}\right)$ be a regular perturbation field. For deriving the formula for the directional derivatives $d J(u ; \Omega)[\mathbf{V}]$ and either for the material derivative $\dot{u}[\mathbf{V}]$ or local derivative $d u[\mathbf{V}]$, we have to construct the one parametric family of mappings $T(t, \mathbf{V})$. Since the expressions for first order derivatives are known to be equivalent, one might use either perturbation of identity $(T(t, \mathbf{V}):=X+t \mathbf{V}(X))$ or the speed method $\left(T(t, \mathbf{V}):=X+\int_{0}^{t} \mathbf{V}(X(s)) d s\right)$.

Without loss of generality, we assume in addition

$$
\mathbf{V}(x)=0, \quad \text { for all } x \in \mathbb{R}^{n}, \quad \text { where } d(x, \bar{S}):=\inf _{y \in S}|x-y| \geq d,
$$

for a certain fixed $d>0$. Consequently, we have in both cases the convenient relation $T(t, \mathbf{V})(\overline{\Omega \cup S}) \equiv \overline{\Omega \cup S}$, for all $t>0, \mathbf{V}$, in particular $T(t, \mathbf{V})\left(\Gamma_{2}\right) \equiv \Gamma_{2}$. In the sequel, we will frequently use the abbreviations

$$
v_{n}:=\left\langle\left.\mathbf{V}\right|_{\Gamma_{1}}, \mathbf{n}\right\rangle, \quad v_{\tau}:=\left\langle\left.\mathbf{V}\right|_{\Gamma_{1}}, \tau\right\rangle .
$$

For algorithmic reliability, one can introduce a description in local coordinates already for the calculus. In particular, for starlike domains with respect to 0 , we denote the unit vector in the outer radial direction by $\mathbf{e}_{r}(\phi)=[\cos \phi, \sin \phi]^{\mathrm{T}}$. Then, we can parameterize the free boundary $\Gamma_{1}$ via polar coordinates $\Gamma_{1}:=\left\{\gamma(\phi)=r(\phi) \mathbf{e}_{r}(\phi)\right.$ : $\phi \in[0,2 \pi]\}$, where $r \in C_{\text {per }}^{3, \alpha}([0,2 \pi])$ is a positive function such that $\operatorname{dist}\left(\Gamma_{1}, \Gamma_{2}\right)>0$ and

$$
C_{\text {per }}^{3, \alpha}([0,2 \pi])=\left\{r \in C^{2, \alpha}([0,2 \pi]): r^{(i)}(0)=r^{(i)}(2 \pi), i=0,1,2,3\right\} .
$$

We consider $d r \in C_{\text {per }}^{3, \alpha}([0,2 \pi])$ as the standard variation for perturbed domains $\Omega_{\varepsilon}$ and boundaries $\Gamma_{1 \varepsilon}$, respectively, defined by $r_{\varepsilon}=r+\varepsilon d r$. Hence, we have $v_{n}=\langle\mathbf{V}, \mathbf{n}\rangle=-d r r / \sqrt{r^{2}+r^{\prime 2}}$ due to $\mathbf{V}=-d r \mathbf{e}_{r}$ and the normal (pointing outward relative to $\Omega$ ) and tangential direction on $\Gamma_{1}$ are given as follows

$$
\mathbf{n}=-\left(r \mathbf{e}_{r}-r^{\prime} \mathbf{e}_{r}^{\prime}\right) \cdot \frac{1}{\sqrt{r^{2}+r^{\prime 2}}}, \quad \tau=\left(r^{\prime} \mathbf{e}_{r}+r \mathbf{e}_{r}^{\prime}\right) \cdot \frac{1}{\sqrt{r^{2}+r^{\prime 2}}} .
$$

Note that by this approach the shape problem is embedded into a Banach space setting. That is, both the shape and its increment, can be viewed as elements of $C_{\text {per }}^{3, \alpha}([0,2 \pi])$. Instead of the unit circle and variations in radial direction, one may use other smooth reference boundaries and fixed variational fields to derive the (explicit) expressions of a second order Fréchet calculus.

Lemma 3.2 The Hadamard representation of the shape gradient reads as follows:

$$
\begin{aligned}
d J(\Omega)[\mathbf{V}]= & \int_{\Gamma_{1}} v_{n}\left\{\left(\langle\nabla u, \tau\rangle-p_{0}\right)\left(\left\langle\nabla^{2} u \cdot \mathbf{n}, \tau\right\rangle-\left\langle\nabla p_{0}, \mathbf{n}\right\rangle\right)\right. \\
& \left.+\frac{1}{2}\left(\langle\nabla u, \tau\rangle-p_{0}\right)^{2} \cdot \kappa+\langle\nabla u, \nabla \lambda\rangle\right\} d \sigma_{x},
\end{aligned}
$$


where $u$ solves (2), $\kappa$ denotes the curvature of $\Gamma_{1}$, and $\lambda$ satisfies the following adjoint equation:

$$
\begin{aligned}
& \Delta \lambda=0, \quad \text { in } \Omega, \\
& \lambda=0, \text { on } \Gamma_{2}, \\
& \frac{\partial \lambda}{\partial \mathbf{n}}=\frac{\partial}{\partial \tau}\left[\frac{\partial u}{\partial \tau}-p_{0}\right], \text { on } \Gamma_{1} .
\end{aligned}
$$

Proof First, the assumptions on regularity of the data and boundaries ensure differentiability, cf. Sokolowski and Zolésio [8]. Hence, a formal differentiation of (1) even in terms of local derivatives is possible and yields immediately

$$
\begin{aligned}
d J(\Omega)[\mathbf{V}]= & \int_{\Gamma_{1}} \frac{1}{2}\left(\frac{\partial u}{\partial \tau}-p_{0}\right)^{2}\{\operatorname{div} \mathbf{V}+\langle D \mathbf{V} \cdot \mathbf{n}, \mathbf{n}\rangle\}+\left(\frac{\partial u}{\partial \tau}-p_{0}\right) \\
& \cdot\left[\left\langle\nabla^{2} u \cdot \mathbf{V}, \tau\right\rangle+\langle\nabla u, d \tau[\mathbf{V}]\rangle-\left\langle\nabla p_{0}, \mathbf{V}\right\rangle+\frac{\partial d u[\mathbf{V}]}{\partial \tau}\right] d \sigma .
\end{aligned}
$$

Here, the local shape derivative $d u=d u[\mathbf{V}]$ reads as

$$
\begin{aligned}
& \Delta d u=0, \quad \text { in } \Omega, \\
& d u=0, \quad \text { on } \Gamma_{2}, \\
& \frac{\partial d u}{\partial \mathbf{n}}=-\operatorname{div}_{\Gamma}\left[v_{n} \nabla_{\Gamma} u\right], \quad \text { on } \Gamma_{1} .
\end{aligned}
$$

Since the shape derivative of the (scaled) normal is always tangential,

$$
d \mathbf{n}[\mathbf{V}]=\left.\left(\frac{\partial}{\partial t} n_{t}\left(T_{t}\right)\right)\right|_{t=0}=-D \mathbf{V}(0)^{*} \cdot \mathbf{n}+\left\langle D \mathbf{V}(0)^{*} \cdot \mathbf{n}, \mathbf{n}\right\rangle \mathbf{n} \perp \mathbf{n},
$$

we conclude $d \tau[\mathbf{V}] \| \mathbf{n}$ for $2 \mathrm{D}$ problems $\left(\tau^{\perp}=\mathbf{n}\right)$. Hence, the expression $\langle\nabla u, d \tau[\mathbf{V}]\rangle$ vanishes due to the boundary condition for $u$.

Next, we transform the first part in (5) by the tangential Green's formula

$$
\begin{aligned}
& \int_{\Gamma} \frac{1}{2}\left(\frac{\partial u}{\partial \tau}-p_{0}\right)^{2} \operatorname{div}_{\Gamma} \mathbf{V} d \sigma \\
& \quad=\int_{\Gamma} \frac{1}{2}\left(\frac{\partial u}{\partial \tau}-p_{0}\right)^{2} \kappa\langle\mathbf{V}, \mathbf{n}\rangle-\left(\frac{\partial u}{\partial \tau}-p_{0}\right)\left\langle\mathbf{V}, \nabla_{\Gamma}\left(\frac{\partial u}{\partial \tau}-p_{0}\right)\right\rangle d \sigma
\end{aligned}
$$

For computing the expression

$$
\left\langle\mathbf{V}, \nabla_{\Gamma}\left(\frac{\partial u}{\partial \tau}\right)\right\rangle
$$

we introduce the oriented distance function $b=b_{\Omega}$, associated with $\Omega$, in a tubular neighborhood 


$$
S_{k}\left(\Gamma_{1}\right):=\left\{x \in \mathbb{R}^{2}: \operatorname{dist}\left(x, \Gamma_{1}\right)<k\right\},
$$

see [7]. Since $\Gamma_{1}$ is compact and (at least) $C^{2}$, there exists an $h>0$ such that $b \in C^{2}\left(S_{2 h}\left(\Gamma_{1}\right)\right)$. Furthermore, its gradient $\nabla b$ is a natural $C^{1}$-extension $\mathcal{N}_{0}:=\nabla b$ for the normal field into a tubular neighborhood $S_{2 h}\left(\Gamma_{1}\right)$ of $\Gamma_{1}$. Due to the relation $\mathbf{n}^{\perp}=-\tau$, the field $-\nabla b^{\perp}$ extends analogously the normalized tangential field in $2 \mathrm{D}$. Moreover, we have the well-known identity

$$
\left.D(\nabla b) \cdot \nabla b\right|_{\Gamma_{1}}=0=\left.D\left(-\nabla b^{\perp}\right) \cdot\left(-\nabla b^{\perp}\right)\right|_{\Gamma_{1}} .
$$

Introducing the projection $P=P(x)$ of a point $x \in S_{2 h}\left(\Gamma_{1}\right)$ by

$$
P(x):=x-b(x) \nabla b(x),
$$

we can define an "intrinsic" extension $\tilde{h} \in C^{1}\left(S_{2 h}\left(\Gamma_{1}\right)\right)$ of an arbitrary function $h \in C^{1}\left(\Gamma_{1}\right)$ as follows:

$$
\tilde{h}(x):=(h \circ P)(x)=h(P(x)), \quad x \in S_{2 h}\left(\Gamma_{1}\right) .
$$

It is well known that this construction provides the property

$$
\left.\nabla(h \circ P)\right|_{\Gamma}=\nabla_{\Gamma} h, \quad \text { on } \Gamma_{1} \text {; }
$$

see [7], Chap. 8, Theorem 5.1. Hence, we compute as follows:

$$
\left\langle\mathbf{V}, \nabla_{\Gamma}\left(\frac{\partial u}{\partial \tau}\right)\right\rangle=\left\langle\mathbf{V}_{\Gamma},\left.\nabla\left(\frac{\widetilde{\partial u}}{\partial \tau}\right)\right|_{\Gamma_{1}}\right\rangle=\left\langle\nabla^{2} u \cdot \mathbf{V}_{\Gamma}, \tau\right\rangle,
$$

where we have taken into account the following relations:

$$
\left.\nabla\left(\widetilde{\frac{\partial u}{\partial \tau}}\right)\right|_{\Gamma_{1}}=\nabla^{2} u \cdot \tau+\left.D\left(-\nabla b^{\perp}\right) \cdot \nabla u\right|_{\Gamma_{1}},
$$

and again, due to the boundary condition for $u$,

$$
\left.\nabla u\right|_{\Gamma_{1}}=\left.\frac{\partial u}{\partial \tau} \cdot \tau \Rightarrow D\left(-\nabla b^{\perp}\right) \cdot \nabla u\right|_{\Gamma_{1}}=\left.\frac{\partial u}{\partial \tau} \cdot D(\nabla b) \cdot \nabla b\right|_{\Gamma_{1}}=0 .
$$

Similarly, we have

$$
\left\langle\nabla p_{0}, \mathbf{V}\right\rangle=v_{n}\left\langle\nabla p_{0}, \mathbf{n}\right\rangle+v_{\tau}\left\langle\nabla p_{0}, \tau\right\rangle=v_{n}\left\langle\nabla p_{0}, \mathbf{n}\right\rangle+\left\langle\mathbf{V}, \nabla_{\Gamma} p_{0}\right\rangle
$$

Consequently, the expression for the shape gradient reads as

$$
\begin{aligned}
d J(\Omega)[\mathbf{V}]= & \int_{\Gamma_{1}} v_{n}\left\{\left(\langle\nabla u, \tau\rangle-p_{0}\right)\left(\left\langle\nabla^{2} u \cdot \mathbf{n}, \tau\right\rangle-\left\langle\nabla p_{0}, \mathbf{n}\right\rangle\right)\right. \\
& \left.+\frac{1}{2}\left(\langle\nabla u, \tau\rangle-p_{0}\right)^{2} \cdot \kappa\right\}+\left(\frac{\partial u}{\partial \tau}-p_{0}\right) \cdot \frac{\partial d u[\mathbf{V}]}{\partial \tau} d \sigma
\end{aligned}
$$


It remains to transform the last part. Integration by parts yields

$$
\begin{aligned}
\int_{\Gamma_{1}}\left(\frac{\partial u}{\partial \tau}-p_{0}\right) \cdot \frac{\partial d u[\mathbf{V}]}{\partial \tau} d \sigma & =\int_{\Gamma_{1}}-\frac{\partial}{\partial \tau}\left(\frac{\partial u}{\partial \tau}-p_{0}\right) \cdot d u[\mathbf{V}] d \sigma \\
& =\int_{\Gamma_{1}}-\frac{\partial \lambda}{\partial \mathbf{n}} \cdot d u[\mathbf{V}] d \sigma
\end{aligned}
$$

Now, we find by the second Green's identity on $\Omega$

$$
\begin{aligned}
0 & =\int_{\Omega} \lambda \Delta d u[\mathbf{V}]-d u[\mathbf{V}] \Delta \lambda d x \\
& =\int_{\Gamma_{1}} \frac{\partial \lambda}{\partial \mathbf{n}} \cdot d u[\mathbf{V}]-\frac{\partial d u[\mathbf{V}]}{\partial \mathbf{n}} \cdot \lambda d \sigma,
\end{aligned}
$$

since related boundary terms on $\Gamma_{2}$ vanish due to the boundary conditions for $\lambda$ and $d u$. Plugging in the related boundary values from (6) and in light of

$$
\operatorname{div}_{\Gamma}\left[v_{n} \nabla_{\Gamma} u\right]=\frac{\partial}{\partial \tau}\left[v_{n} \frac{\partial u}{\partial \tau}\right]
$$

we arrive at

$$
\int_{\Gamma_{1}}\left(\frac{\partial u}{\partial \tau}-p_{0}\right) \cdot \frac{\partial d u[\mathbf{V}]}{\partial \tau} d \sigma=\int_{\Gamma_{1}} \frac{\partial}{\partial \tau}\left[v_{n} \frac{\partial u}{\partial \tau}\right] \cdot \lambda d \sigma .
$$

Finally, we obtain the Hadamard representation (3) by a further integration by parts and by again making use of the boundary condition for the state $u\left(\partial u /\left.\partial \mathbf{n}\right|_{\Gamma_{1}}=0\right)$.

Remark 3.1 According to the Hadamard structure theorem (see [7, 9]), formula (3) represents a regular scalar distribution $\mathcal{G}(\Gamma) \in C^{3, \alpha}\left(\Gamma_{1}\right)^{*}$,

$$
d J(\Omega)[\mathbf{V}]=\left\langle\mathcal{G}(\Gamma), v_{n}\right\rangle_{C^{k}\left(\Gamma_{1}\right)}=\int_{\Gamma_{1}} G_{\Gamma}(x) \cdot v_{n}(x) d \sigma, \quad \mathbf{V} \in C^{k}\left(\mathbb{R}^{n}, \mathbb{R}^{n}\right),
$$

where

$$
\begin{aligned}
G_{\Gamma}(x):= & \left\{\left(\langle\nabla u, \tau\rangle-p_{0}\right)\left(\left\langle\nabla^{2} u \cdot \mathbf{n}, \tau\right\rangle-\left\langle\nabla p_{0}, \mathbf{n}\right\rangle\right)\right. \\
& \left.+\frac{1}{2}\left(\langle\nabla u, \tau\rangle-p_{0}\right)^{2} \cdot \kappa+\langle\nabla u, \nabla \lambda\rangle\right\}(x), \quad x \in \Gamma_{1} .
\end{aligned}
$$

Sometimes in the literature, the function $G_{\Gamma}$ is referred to as the shape gradient, too, and it is proposed using $G_{\Gamma}$ directly as an update for the boundary. However, we have $G_{\Gamma} \in C^{1, \alpha}\left(\Gamma_{1}\right)$ for $\Gamma_{1} \in C^{3, \alpha}$. Moreover, this loss of regularity is generic, i.e., $\Gamma_{1} \in C^{k, \alpha}$ implies $G_{\Gamma} \in C^{k-2, \alpha}\left(\Gamma_{1}\right)$ for arbitrary $k>3$.

We continue with an immediate consequence of the Hadamard representation. 
Lemma 3.3 (Necessary condition) Let the domain $\Omega^{\star}$ be such that the state $u=$ $u\left(\Omega^{\star}\right)$ matches perfectly the prescribed pressure distribution on $\Gamma_{1}$, i.e., there holds

$$
\langle\nabla u, \tau\rangle=p_{0}, \quad \text { on } \Gamma^{\star} .
$$

Then, the domain $\Omega^{\star}$ fulfills the necessary optimality condition

$$
d J\left(\Omega^{\star}\right)[d r]=0 \quad \text { for all } d r \in C_{\text {per }}^{3, \alpha}([0,2 \pi]) .
$$

Proof Using (8), we conclude from (4) that $\lambda^{*}=\lambda\left(\Omega^{\star}\right) \equiv 0$ on $\Omega^{\star}$. Consequently, it follows that

$$
\left(\frac{\partial u}{\partial \tau}-p_{0}\right)\left(\frac{\partial^{2} u}{\partial \mathbf{n} \partial \tau}-\frac{\partial p_{0}}{\partial \mathbf{n}}\right)+\frac{\kappa}{2}\left(\frac{\partial u}{\partial \tau}-p_{0}\right)^{2}+\langle\nabla \lambda, \nabla u\rangle \equiv 0, \quad \text { on } \Gamma_{1}^{*},
$$

which, in view of (3), implies the assertion.

Remark 3.2 We emphasize that solutions of the necessary condition (9) may exist that do not satisfy the matching condition (8) for the pressure data. However, only domains $\Omega^{\star}$ which provide perfectly matching data correspond to global minima since $J\left(\Omega^{\star}\right)=0$.

Remark 3.3 In terms of polar coordinates $(d J(\Omega)[\mathbf{V}] \widehat{=} d J(\Omega)[d r])$, the related representation reads as

$$
\begin{aligned}
d J(\Omega)[d r]= & \int_{0}^{2 \pi} d r r\left\{\left(\frac{\partial u}{\partial \tau}-p_{0}\right) \cdot\left(\frac{\partial^{2} u}{\partial \mathbf{n} \partial \tau}-\frac{\partial p_{0}}{\partial \mathbf{n}}\right)\right. \\
& \left.+\frac{1}{2}\left(\langle\nabla u, \tau\rangle-p_{0}\right)^{2} \cdot \frac{r^{2}+2 r^{\prime 2}-r r^{\prime \prime}}{{\sqrt{r^{2}+r^{\prime 2}}}^{3}}+\langle\nabla \lambda, \nabla u\rangle\right\} d \phi .
\end{aligned}
$$

Moreover, direct calculations may recover more explicitly the general transformations from the proof of Lemma 3.2.

\subsection{Shape Hessian Representation in Local Coordinates}

For the derivation of the shape Hessian representation in local coordinates, we recall a technical result from [12]. It concerns a particular case of (7), useful for explicit calculations.

Lemma 3.4 Let $\Omega \subset \mathbb{R}^{2}$ be a domain with boundary $\Gamma \in C^{2}$ and $\left.\mathbf{V}\right|_{\Gamma} \in C^{1}(\Gamma)$. Then, the shape derivatives of the scaled normal and tangential field read as follows:

$$
\begin{aligned}
& d \mathbf{n}[\mathbf{V}]=-\left\langle\mathbf{n}, \frac{d}{d s}\left(\left.\mathbf{V}\right|_{\Gamma}\right)\right\rangle \cdot \tau, \\
& d \tau[\mathbf{V}]=\left\langle\mathbf{n}, \frac{d}{d s}\left(\left.\mathbf{V}\right|_{\Gamma}\right)\right\rangle \cdot \mathbf{n},
\end{aligned}
$$

where $\frac{d}{d s}$ denotes differentiation with respect to the arclength. 
Since the local shape derivative $d \lambda$ of the adjoint state $\lambda$ will appear in the shape Hessian representation, we continue with the computation of the related characterization equation.

Lemma 3.5 Let the regularity assumptions hold on $\Omega$ and on the problem data stated at the beginning of Sect. 3.1. Then, the local shape derivative $d \lambda=d \lambda[\mathbf{V}]$ exists and is a solution of the following boundary value problem:

$\Delta d \lambda=0, \quad$ in $\Omega$,

$d \lambda=0, \quad$ on $\Gamma_{2}$,

$$
\begin{aligned}
\frac{\partial d \lambda}{\partial \mathbf{n}}= & \left\langle\nabla^{2} \lambda \cdot \mathbf{V}, \mathbf{n}\right\rangle+\frac{\partial \lambda}{\partial \tau} \cdot\left\langle\mathbf{n}, \frac{d}{d s}\left(\left.\mathbf{V}\right|_{\Gamma}\right)\right\rangle \\
& +\left\langle\nabla^{3} u \cdot \mathbf{V} \cdot \tau, \tau\right\rangle+\left\langle\nabla^{2} d u[\mathbf{V}] \cdot \tau, \tau\right\rangle \\
& +2\left\langle\mathbf{n}, \frac{d}{d s}\left(\left.\mathbf{V}\right|_{\Gamma}\right)\right\rangle \cdot \frac{\partial^{2} u}{\partial \mathbf{n} \partial \tau}-\left\langle\nabla^{2} p_{0} \cdot \mathbf{V}, \mathbf{n}\right\rangle+\frac{\partial p_{0}}{\partial \mathbf{n}} \cdot\left\langle\mathbf{n}, \frac{d}{d s}\left(\left.\mathbf{V}\right|_{\Gamma}\right)\right\rangle, \text { on } \Gamma_{1} .
\end{aligned}
$$

Proof By the regularity of our data, we have $\lambda \in C^{2, \alpha}(\bar{\Omega})$, and the existence of the material derivative $\dot{\lambda}[\mathbf{V}] \in C^{1, \alpha}(\bar{\Omega})$ follows by standard arguments; see [7, 8]. Moreover, the regularity $d \lambda[\mathbf{V}] \in C^{1, \alpha}(\bar{\Omega})$ is provided by the identity

$$
\dot{\lambda}[\mathbf{V}]=d \lambda[\mathbf{V}]+\langle\mathbf{V}, \nabla \lambda\rangle \text {. }
$$

Hence, the boundary condition for $d \lambda$ on $\Gamma_{1}$ can be obtained by computing directly the shape derivative

$$
d\{\langle\nabla \lambda, \mathbf{n}\rangle\}[\mathbf{V}]=\langle\nabla d \lambda, \mathbf{n}\rangle+\left\langle\nabla^{2} \lambda \cdot \mathbf{V}, \mathbf{n}\right\rangle-\langle\nabla \lambda, \tau\rangle \cdot\left\langle\mathbf{n}, \frac{d}{d s}\left(\left.\mathbf{V}\right|_{\Gamma}\right)\right\rangle,
$$

and similarly for the right-hand side of the related boundary condition in (4).

We shall now derive the shape Hessian representation in terms of polar coordinates. To avoid inconvenient shape differentiation of geometric terms like curvature and normal or tangential fields as much as possible, we start from an "intermediate expression" for the shape gradient,

$$
\begin{aligned}
d J(\Omega)[d r]= & \int_{0}^{2 \pi} \frac{r d r+r^{\prime} d r^{\prime}}{\sqrt{r^{2}+r^{\prime 2}}} \cdot \frac{\left(\langle\nabla u, \tau\rangle-p_{0}\right)^{2}}{2}+d r r\langle\nabla \lambda, \nabla u\rangle d \phi \\
& +\int_{0}^{2 \pi} d r\left(\langle\nabla u, \tau\rangle-p_{0}\right)\left[\left\langle\nabla^{2} u \cdot \mathbf{e}_{r}, \tau\right\rangle+\left\langle\nabla p_{0}, \mathbf{e}_{r}\right\rangle\right] \sqrt{r^{2}+r^{\prime 2}} d \phi .
\end{aligned}
$$

Remark 3.4 Of course, (13) is equivalent to (10). In particular, the explicit dependence from local (or material) shape derivatives of the state is already removed. In 
two-dimensional problems, polar coordinates are convenient. For applications in arbitrary dimension, the parameterization by a reference manifold $\Gamma_{0} \in C^{k+1, \alpha}$ might be more convenient. We introduce a scalar parameterization $\rho \in C^{k, \alpha}\left(\Gamma_{0}\right)$. The shape (or boundary) variations are defined via the normal $\mathbf{n}_{0}$, and similarly the variations on the perturbed boundaries,

$$
\Gamma_{1}^{t}=\Gamma_{0}+t \rho \mathbf{n}_{0}, \quad d \Gamma_{1}^{t}[\nu]:=v \circ T_{t \rho} \cdot \mathbf{n}_{0} \circ T_{t \rho} .
$$

In any case, the structure of the essential terms of the shape Hessian remains unaffected from the particular choice.

To keep the subsequent expression of the shape Hessian readable, we introduce the following abbreviations:

$$
\begin{aligned}
j\left(u, \tau, p_{0}\right):= & \langle\nabla u, \tau\rangle-p_{0}, \\
d j\left(u, \tau, p_{0}\right)[d r]= & d r \cdot\left[\left\langle\nabla^{2} u \cdot \mathbf{e}_{r}, \tau\right\rangle-\left\langle\nabla p_{0}, \mathbf{e}_{r}\right\rangle\right], \\
d^{2} j\left(u, \tau, p_{0}\right)\left[d r_{1}, d r_{2}\right]= & d r_{1} d r_{2} \cdot\left[\left\langle\nabla^{3} u \cdot \mathbf{e}_{r} \cdot \mathbf{e}_{r}, \tau\right\rangle-\left\langle\nabla^{2} p_{0} \cdot \mathbf{e}_{r}, \mathbf{e}_{r}\right\rangle\right] \\
& +d r_{1} \cdot\left[\left\langle\nabla^{2} d u\left[d r_{2}\right] \cdot \mathbf{e}_{r}, \tau\right\rangle\right. \\
& \left.+\left\langle\nabla^{2} u \cdot \mathbf{e}_{r}, \mathbf{n}\right\rangle\left\langle\mathbf{n}, \frac{d\left(d r_{2} \mathbf{e}_{r}\right)}{d s}\right\rangle\right] \\
d(\langle\nabla u, \nabla \lambda\rangle)[d r]= & \langle\nabla d u[d r], \nabla \lambda\rangle+\langle\nabla u, \nabla d \lambda[d r]\rangle \\
& +d r\left\{\left\langle\nabla^{2} u \cdot \mathbf{e}_{r}, \nabla \lambda\right\rangle+\left\langle\nabla u, \nabla^{2} \lambda \cdot \mathbf{e}_{r}\right\rangle\right\},
\end{aligned}
$$

where we used again in the second (and third) expression the identity

$$
\langle\nabla u, d \tau[d r]\rangle=\langle\nabla u, \mathbf{n}\rangle \cdot\left\langle\mathbf{n}, \frac{d}{d s}\left(d r \mathbf{e}_{r}\right)\right\rangle=0 .
$$

Lemma 3.6 Under the regularity assumptions stated in Sect. 3.1, the objective $J$ is twice shape differentiable and the shape Hessian reads as follows:

$$
\begin{aligned}
& d^{2} J(\Omega)\left[d r_{1}, d r_{2}\right] \\
& =\int_{0}^{2 \pi} d r_{1} d r_{2}\langle\nabla \lambda, \nabla u\rangle+r d r_{1} d(\langle\nabla u, \nabla \lambda\rangle)\left[d r_{2}\right] d \phi \\
& \quad+\int_{0}^{2 \pi}\left\{d j\left(u, \tau, p_{0}\right)\left[d r_{1}\right]+d j\left(u, \tau, p_{0}\right)\left[d r_{2}\right]\right\} j\left(u, \tau, p_{0}\right) \sqrt{r^{2}+r^{\prime 2}} \\
& \quad+\left\{d j\left(u, \tau, p_{0}\right)\left[d r_{1}\right] \frac{r d r_{2}+r^{\prime} d r_{2}^{\prime}}{\sqrt{r^{2}+r^{\prime 2}}}\right. \\
& \left.\quad+d j\left(u, \tau, p_{0}\right)\left[d r_{2}\right] \frac{r d r_{1}+r^{\prime} d r_{2}^{\prime}}{\sqrt{r^{2}+r^{\prime 2}}}\right\} j\left(u, \tau, p_{0}\right)
\end{aligned}
$$




$$
\begin{aligned}
& +\frac{\left(j\left(u, \tau, p_{0}\right)^{2}\right.}{2} \cdot \frac{\left(r d r_{1}^{\prime}-r^{\prime} d r_{1}\right)\left(r d r_{2}^{\prime}-r^{\prime} d r_{2}\right)}{\sqrt{r^{2}+r^{\prime 2}}} \\
& +d j\left(u, \tau, p_{0}\right)\left[d r_{1}\right] d j\left(u, \tau, p_{0}\right)\left[d r_{2}\right] \sqrt{r^{2}+r^{\prime 2}} \\
& +j\left(u, \tau, p_{0}\right) d^{2} j\left(u, \tau, p_{0}\right)\left[d r_{1}, d r_{2}\right] \sqrt{r^{2}+r^{\prime 2}} d \phi .
\end{aligned}
$$

Remark 3.5 In practice, the prescribed pressure data $p_{0}$ will be physically relevant. That is, the current and target pressure distributions will be a close match, resulting in

$$
j^{k}\left(u, \tau, p_{0}\right):=j\left(\Gamma_{1}^{k} ; u\left(\Omega^{k}\right), \tau^{k}, p_{0}\right) \approx 0,
$$

at least for $k$ sufficiently large. Hence, terms with leading $j\left(u, \tau, p_{0}\right)\left(\right.$ or $\left.j\left(u, \tau, p_{0}\right)^{2}\right)$ can be ignored for preconditioning procedures, see the next section. In particular, several terms disappear, where the general nature is not obvious due to representation in polar coordinates.

Remark 3.6 For convenience, we briefly summarize known facts from the literature [10-14]. Namely, let us assume, that all shapes $\Omega$ (i.e., $\Gamma_{1}$ ) of interest, which are of equal regularity, can be identified uniquely with the associated elements of a function space $C^{k, \alpha}\left(\Gamma_{1}^{0}\right)$, at least locally around a reference shape $\Omega_{0}$,

$$
U_{\delta}\left(\Omega_{0}\right) \ni \Omega \quad \Leftrightarrow \quad x \in U_{\rho}\left(x_{0}\right) \subset X=C^{k, \alpha}\left(\Gamma_{1}^{0}\right), \quad U_{\delta}\left(\Omega_{0}\right) \subset C^{k, \alpha} .
$$

If the objective is twice Fréchet differentiable, the shape gradient is from the dual space, $\nabla J(x) \in X^{*}$, and the Hessian defines a linear continuous operator, $\nabla^{2} J(x) \in$ $L\left(X, X^{*}\right)$, as well as a continuous bilinear form on $X$,

$$
\left|\nabla^{2} J(r)\left[d x_{1}, d x_{2}\right]\right| \leq C\left\|d x_{1}\right\|_{X}\left\|d x_{2}\right\|_{X}, \quad \text { uniformly for all } x \in U_{\rho}\left(x_{0}\right) .
$$

However, for integral functionals and due to the boundary integral representation of the shape Hessian, the induced bilinear form continuously extends to a bilinear form on $H^{s}\left(\Gamma_{1}^{0}\right)$, for a certain $s \geq 0$. Consequently, the shape Hessian defines a pseudodifferential operator of order $2 s$. Moreover, coercivity of the shape Hessian in $H^{s}$,

$$
\nabla^{2} J(x)[d x, d x] \geq c_{0}\|d x\|_{H^{s}}, \quad c_{0}>0,
$$

will ensure optimality, provided a certain refined second order remainder estimate is satisfied. Further, it determines the Sobolev space for the "resmoothing" procedure, described in the next section. We skip further details for the problem under consideration, since this will be the topic of a forthcoming paper.

\section{Steepest Descent Method Revisited}

We employ a preconditioned steepest descend method. In the engineering literature, this is also often called a "Sobolev-Gradient Method", while mathematically, it can be thought of as an approximative SQP method. We define the gradient as the Rieszrepresentation of the derivative. Thus, a scalar product which is close to the one induced by the reduced Hessian operator will result in a much faster convergence, while also preventing the loss of regularity. 
Definition 4.1 For a differentiable function $f: H \rightarrow \mathbb{R}$, where $H$ is a Hilbert space with scalar product $(.,)_{H}$, we define the gradient as the Riesz-representation of the derivative $f^{\prime}$, such that

$$
(\nabla f, v)_{H}=f^{\prime}(v), \quad \forall v \in H,
$$

where $f^{\prime}$ is defined by the linear operator

$$
f^{\prime}(q):\left.\quad v \mapsto \frac{d}{d t}\right|_{t=0} f(q+t \cdot v), \quad \forall v \in H .
$$

Note that the computation of a meaningful shape Hessian usually requires a global Hilbert space embedding. Otherwise, the Hessian will depend on the deformation, i.e. using perturbation of identity or the speed method will result in different expressions for the shape Hessian.

Let $\nabla J(q)$ denote the gradient with respect to the standard $L_{2}$ scalar product and let $\nabla_{S} J(q)$ denote the gradient with respect to some other "smoother" inner product. If the smoother inner product of $H^{1}(X)$ is given by

$$
\langle a, b\rangle_{H^{1}}=\langle M a, b\rangle_{L^{2}}
$$

for an appropriate positive definite operator $M: L^{2}(X) \rightarrow L^{2}(X)$, then we arrive at the following relation of the canonical and the noncanonical gradients:

$$
\begin{aligned}
D J(q) h & =\left\langle\nabla_{S} J(q), h\right\rangle_{H^{1}} \\
& =\left\langle M \nabla_{S} J(q), h\right\rangle_{L^{2}}=\langle\nabla J(q), h\rangle_{L^{2}},
\end{aligned}
$$

which results in

$$
\nabla_{S} J(q)=M^{-1} \nabla J(q) .
$$

Thus choosing $M$ as the reduced Hessian operator turns the Sobolev steepest descent into Newton's iteration. However, the proper choice of the Sobolev space heavily depends on the true nature of the shape Hessian, cf. Remark 3.6.

\subsection{Preconditioning}

A crucial aspect of the preconditioning is the identification of the proper (pseudo-) differential operator nature of the terms $\nabla d \lambda[\mathbf{V}]$ and $\nabla d u[\mathbf{V}]$. A Fourier analysis similar to [3, 4, 15] shows $\nabla d \lambda[\mathbf{V}] \approx \frac{\partial^{2} \mathbf{V}}{\partial \tau^{2}}$ and $\nabla d u[\mathbf{V}] \approx \frac{\partial \mathbf{V}}{\partial \tau}$ on the wing, which means that the reduced Hessian as described by Lemma 3.6 acts like a pseudodifferential operator of order +2 . More details can be found in a forthcoming paper. Thus, the approximation of this Hessian has to have the following coarse structure:

$$
H \approx-\frac{\partial}{\partial \tau}\left(k \frac{\partial}{\partial \tau}\right),
$$

or with the addition of a constant 0th order term:

$$
H \approx-\frac{\partial}{\partial \tau}\left(k \frac{\partial}{\partial \tau}\right)+i d,
$$


where $k$ is a smoothing parameter and id is the identity operator. Several authors have used a similar basic layout with much success. Gherman and Schulz [16] also used Fourier analysis to determine $k$, while others have interpreted $k$ as a constant parameter in the optimization algorithm and then tried to find the best value by automatic means, see [17]. However, thanks to Lemma 3.6 and Remark 3.5, we can take far more information into account than just the highest order. Let us mention further, that for somewhat simpler problems, the Newton method is investigated in several papers by implementing the whole shape Hessian. In particular, cubic B-splines are used as ansatz functions for the shape by Eppler, Harbrecht and Schneider [14], to verify theoretically predicted orders of convergence. Another possibility is to study the shape Hessian via sinusoidal perturbations of the annulus as presented in [5], since a Fourier series ansatz provide exponential approximation in case of optimal domains with analytic boundary $[18,19]$.

Although most - if not all-2D airfoils are indeed starshaped domains, we do not want to restrict our practical implementation to such domains, especially since complex 3D aircrafts are not starshaped. We therefore choose to perturb every surface node in normal direction only: $\mathbf{V}=\alpha n$, cf. Remark 3.4. Unfortunately, this makes carrying over all parts of the Hessian for a starshaped domain to a normal perturbation impossible. However, certain parts can still be identified:

As mentioned above, the term $\langle\nabla u, \nabla d \lambda[]$.$\rangle is the term that adds the order +2$ to the Hessian, meaning $\nabla d \lambda[.] \approx \frac{\partial^{2}}{\partial \tau^{2}}$. And therefore, the expression $\langle\nabla u, \nabla d \lambda[$.] $]\rangle$ suggests using the approximation $k \approx \nabla u=\frac{\partial u}{\partial \tau}$ due to the boundary conditions. When further assuming that the derivative of the state variation $\nabla d u\left[\right.$.] $\approx \frac{\partial}{\partial \tau}$ is of first order, then the term $\langle\nabla d u[],. \nabla \lambda\rangle$ suggests adding $\frac{\partial \lambda}{\partial \tau} \frac{\partial}{\partial \tau}$ to the preconditioner.

Finally, the part $\langle\nabla \lambda, \nabla u\rangle$ of the Hessian, which does not contain any derivatives, can simply be read, and when putting everything together, one arrives at the following preconditioning ODE to be solved for $\nabla_{S} J$ :

$$
-\frac{\partial}{\partial \tau}\left[k\left(\frac{\partial}{\partial \tau} \nabla_{S} J\right)\right]+\frac{\partial \lambda}{\partial \tau}\left(\frac{\partial}{\partial \tau} \nabla_{S} J\right)+\frac{\partial \lambda}{\partial \tau} \frac{\partial u}{\partial \tau} \nabla_{S} J=\nabla J,
$$

where again $\nabla_{S} J$ denotes the new "smoother" gradient and $\nabla J$ is the canonical $L_{2}$ gradient and $k \approx \frac{\partial u}{\partial \tau}=\langle\nabla u, \tau\rangle$ is related to the tangential velocity.

\section{Numerical Results}

\subsection{Discretization of the State Equation}

For our optimizations, the state equation is discretized by an "aerodynamic panel solver", which is similar to a boundary element method. Using Green's 2nd Identity, the Laplace equation for the volume can be transformed into an integral equation on the boundary of the wing. Similar to finite elements, the potential $u$ is discretized as a finite linear combination of ansatz functions, resulting in a small, dense, and linear system to be solved. Any additional boundary conditions for the free stream velocity on $\Gamma_{2}$ are embedded in the ansatz functions. To further ensure a physically 
meaningful solution and to allow a proper prediction of the lift, the trailing edge stagnation point is prescribed by a slightly modified boundary condition, thus introducing lift via circulation similar to the Kutta-Joukowski theorem. For further information about the solver, the aerodynamics, and on how to ensure a physical solution that matches experimental measurements, we would like to refer to [20]. To avoid any discrepancies with the "analytical adjoint" equation as derived above, the adjoint of the state equation was taken as the transpose of the discrete linear state equation, i.e. adjoint in $\mathbb{R}^{n}$ with the standard scalar product.

\subsection{Optimization}

The aim of this section is to compare the performance of the different preconditioners in practical applications

- when using no preconditioner at all,

- when using (14) as a preconditioner,

- when using (15) as a preconditioner,

- when using the extended 2nd, 1st, and 0th order preconditioner (16).

We are both interested in a comparison of the performance of the different preconditioners to each other, but also with respect to the number of design parameters. Our aim is to match the pressure distributions generated by different NACA 4 digit airfoils. That means we start at the symmetrical NACA0012 airfoil and wish to match the pressure distribution generated by the cambered NACA4412 profile. Figure 2 illustrates this. A detailed description of the NACA shapes can be found in [21]. We again would like to stress out, that we are not using the NACA digits as design parameter but the mesh nodes. Our optimization method is the preconditioned steepest descent with Armijo linesearch.

\subsection{Comparison of Different Smoothers}

Our first series of tests are meant to compare the different smoothers. We start with a comparison of the results when no preconditioner is used with those of the "standard" 2nd order smoother (15). The history of the objective function is shown in Fig. 3. Without any smoothing, the resulting degeneration of the shape does not allow any convergence at all, while the second order smoother immediately ensures a smooth

Fig. 2 Initial NACA0012 airfoil and target NACA4412 airfoil
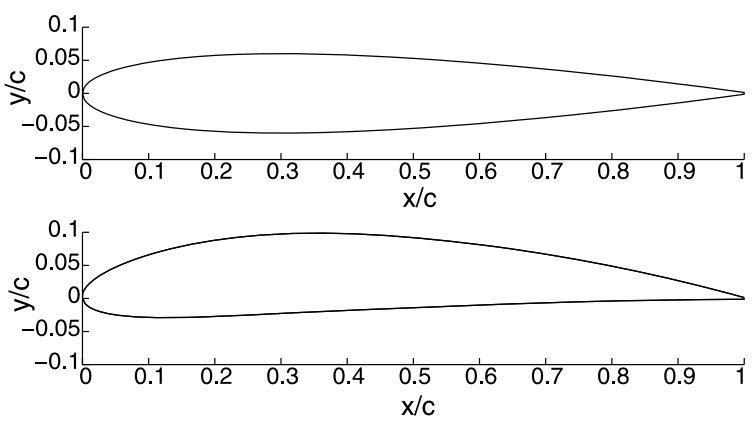
shape over all iterations. Using the tangential velocity in (15) is always better than any attempts to find a constant value for $k$ by hand. Figure 4 shows the resulting optimal shapes. The dotted line is the target pressure distribution. The next series of tests is conducted on non-symmetric airfoils, and hence are tests with aerodynamic lift.

Fig. 3 Optimization history, symmetric airfoils, smoothed by $k=\sqrt{\langle\nabla u, \tau\rangle+\delta}$

Fig. 4 Optimal nonlifting shapes and their pressure distribution without and with smoothing
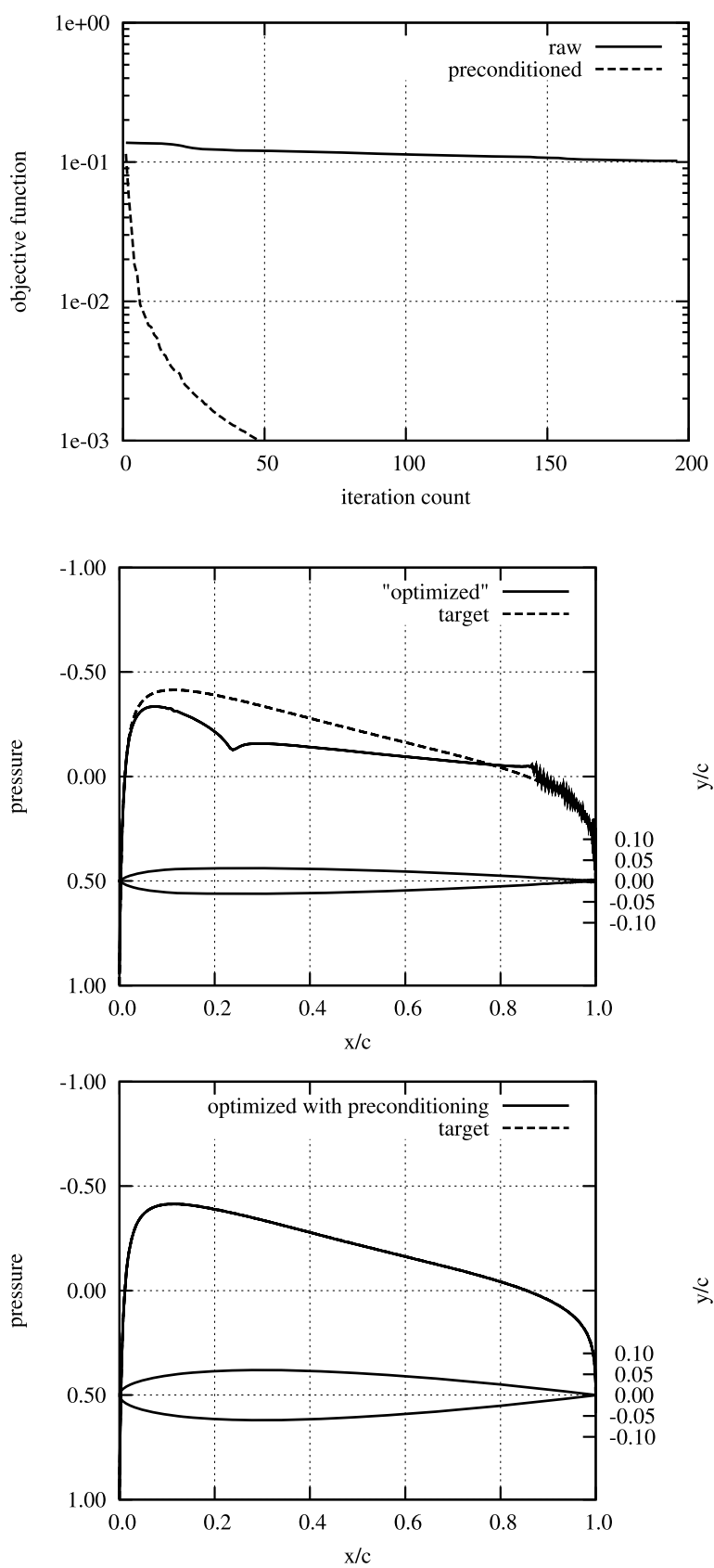
Fig. 5 Optimization history, lifting airfoils. Smoothing proportional to discretization

Fig. 6 Optimal lifting shapes without and with smoothing
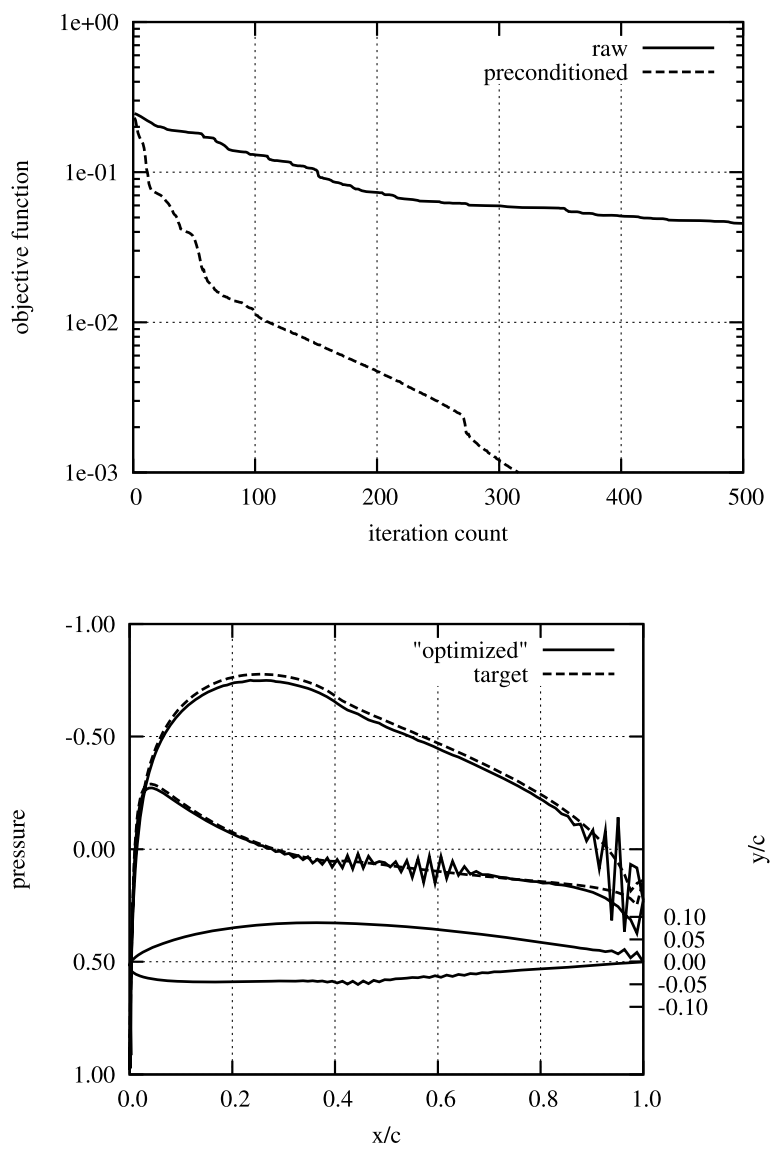

$\frac{0}{\lambda}$

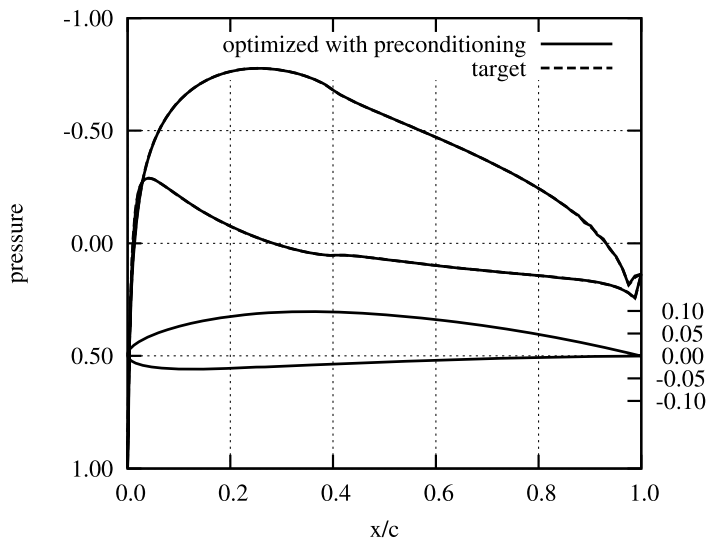

$\lesssim$

Unsurprisingly, the results basically repeat itself: Loss of regularity without smoothing which is immediately cured by the smoother (15). Furthermore, we test the length of each line segment, i.e. "panel", of the surface discretization as a value for $k$. The results are shown in Fig. 5, and the optimal shapes are presented in Fig. 6. 


\subsubsection{Comparisons with Respect to the Number of Design Parameters}

We now focus on studies dealing with the relation of the number of design parameters and the smoother used, and here our custom smoother (16) seems most promising. All tests are conducted for the nonlifting, symmetric case. Table 1 provides the number of smoothed gradient steps needed to achieve the stopping criteria of $10^{-8}$. The numbers in the header refer to the respective preconditioner. For the smoothing parameter we always chose $k=\sqrt{\langle\nabla u, \tau\rangle^{2}+\delta}$, where $\delta$ is a small positive number preventing preconditioner (14) from becoming singular at points with no tangential velocity. The column "Factor" shows the speedup of our custom shape Hessian based preconditioner (16) over the next best preconditioner. Note that the preconditioner (14) breaks down after slightly more than 256 design parameters, as the Armijo rule no longer provides a meaningful step length due to preconditioner (14) not being coercive. One can see, that the shape Hessian based preconditioner (16) can be almost three times faster than the "standard" smoothers.

Another property of these preconditioners - especially preconditioner (16) - is the fact, that the level independent convergence rate of the Newton method is more or less preserved, meaning the number of preconditioned gradient steps growths not faster than logarithmic when the number of design parameters is increased. To illustrate this, we push preconditioner (16) to the limit by increasing the design parameters until we reach a total of 2000 . The resulting curve is shown in Fig. 7. Note that in order to keep the computational time manageable, the stopping criteria is somewhat relaxed, resulting in the fewer steps when comparing Fig. 7 with Table 1.

Table 1 Performance

\begin{tabular}{lllll}
\hline Design parameter & $(14)$ & $(15)$ & $(16)$ & Factor \\
\hline 64 & 150 & 182 & 82 & 1.83 \\
128 & 245 & 238 & 85 & 2.80 \\
256 & 304 & 317 & 136 & 2.24 \\
512 & - & 342 & 163 & 2.10 \\
1024 & - & 383 & 224 & 1.71 \\
\hline
\end{tabular}

Fig. 7 Growth of the smoothed gradient steps compared to the number of design variables

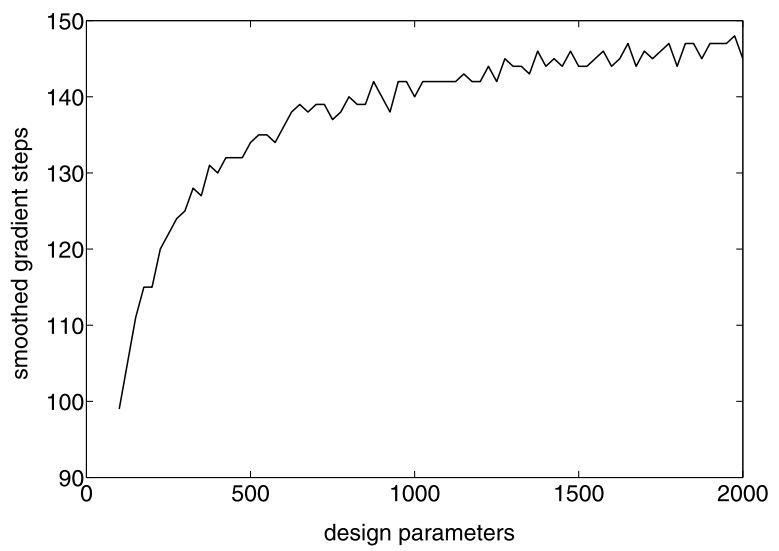




\section{Conclusions}

The potential flow pressure matching is a classical inverse design aerodynamic problem. The resulting loss of regularity during the optimization poses challenges for shape optimization with normal perturbation of the surface mesh nodes. Apparently, smoothness need not be enforced by the parameterization but depends only on the scalar product used to determine the gradient.

Shape calculus is used to arrive at a Hadamard form of the shape gradient which is totally independent of the number of design parameters and does not need any "mesh sensitivities", which are often silently carried out but not mentioned in present aerodynamic design endeavors. The surface integral of the objective function and the high dependence on objects linked to the current shape-like the normal and tangent-make the derivation of Hadamard Gradient non-trivial.

The shape Hessian is derived in local coordinates for a starshaped domain. Significant parts of Hessian are identified and combined with an aerodynamic panel solver. The resulting shape Hessian preconditioner is shown to be up to 2.8 times faster than "standard" 2nd order only preconditioners. Additionally, preconditioning gives potential for level independent convergence, meaning that the number of preconditioned gradient steps grows at most logarithmically with the number of design parameters.

\section{References}

1. Gauger, N.R.: Das Adjungiertenverfahren in der aerodynamischen Formoptimierung. Forschungsbericht Deutsches Zentrum für Luft- und Raumfahrt e.V. (2003)

2. Jameson, A.: Aerodynamic design via control theory. J. Sci. Comput. 3, 23-260 (1988)

3. Arian, E., Ta'asan, S.: Analysis of the Hessian for aerodynamic optimization: Inviscid flow. Technical report, 96-28, Institute for Computer Applications in Science and Engineering (1996)

4. Arian, E., Vatsa, V.N.: A preconditioning method for shape optimization governed by the Euler equations. Technical report, 98-14, Institute for Computer Applications in Science and Engineering (1998)

5. Eppler, K., Harbrecht, H.: A regularized Newton method in electrical impedance tomography using shape Hessian information. Control Cybern. 34(1) (2005)

6. Jameson, A.: Future research avenues in computational engineering and design. In: Ball, J.M., Hunt, J.C.R. (eds.) Proceedings of the Fourth International Congress on Industrial and Applied Mathematics. Oxford University Press, New York (2000)

7. Delfour, M., Zolésio, J.-P.: Shapes and Geometries. SIAM, Philadelphia (2001)

8. Sokolowski, J., Zolésio, J.-P.: Introduction to Shape Optimization. Springer, Berlin (1992)

9. Delfour, M., Zolésio, J.-P.: Velocity method and Lagrangian formulation for the computation of the shape Hessian. SIAM Control Optim. 29, 1414-1442 (1991)

10. Dambrine, M., Pierre, M.: About stability of equilibrium shapes. Math. Model. Numer. Anal. 34, 811-834 (2000)

11. Dambrine, M.: On variations of the shape Hessian and sufficient conditions for the stability of critical shapes. Rev. R. Acad. Cienc. Ser. A Mat. 96(1), 95-121 (2002)

12. Eppler, K.: Second derivatives and sufficient optimality conditions for shape functionals. Control Cybern. 29, 485-512 (2000)

13. Eppler, K.: Boundary integral representations of second derivatives in shape optimization. Discuss. Math. Differ. Inclusion Control Optim. 20, 63-78 (2000)

14. Eppler, K., Harbrecht, H., Schneider, R.: On Convergence in Elliptic Shape Optimization. SIAM J. Control Optim. 45, 61-83 (2007)

15. Schmidt, S., Schulz, V.: Impulse response approximations of discrete shape Hessians with application in CFD. DFG-preprint, SPP1253-10-02 (2008)

16. Gherman, I., Schulz, V.: Preconditioning of one-shot pseudo-timestepping methods for shape optimization. Proc. Appl. Math. Mech. 5(1), 741-742 (2005) 
17. Kim, S., Hosseini, K., Leoviriyakit, K., Jameson, A.: Enhancement of adjoint design methods via optimization of adjoint parameters. Presented at the 43rd AIAA Aerospace Sciences Meeting and Exhibit, Reno, NV, 10-13 January 2005

18. Eppler, K., Harbrecht, H.: Numerical solution of elliptic shape optimization problems using waveletbased BEM. Optim. Methods Softw. 18, 105-123 (2003)

19. Eppler, K., Harbrecht, H.: 2nd order shape optimization using wavelet BEM. Optim. Methods Softw. 21, 135-153 (2006)

20. Katz, J., Plotkin, A.: Low-Speed Aerodynamics-From Wing Theory to Panel Models. Int. edn. McGraw-Hill, New York (1991)

21. Jacobs, E.N., Ward, K.E., Pinkerton, R.M.: The characteristics of 78 related airfoil sections from tests in the variable-density wind tunnel. NACA report No. 460 (1933) 